\title{
A New Method for Virtual Machine Cluster Scheduling
}

\author{
Yu Shuchun, Qiu Lin and Liu Shuang \\ The higher educational key laboratory for Measuring \& Control Technology and \\ Instrumentations of Heilongjiang province, Harbin University of Science and \\ Technology, Harbin, China \\ yscls2013@163.com
}

\begin{abstract}
Aiming at the reasonable configuration of virtual cluster and physical host, a new virtual machine cluster scheduling method is proposed. First of all, according to the resource requirements of the task, select the resource conditions similar to the virtual machine cluster. In the clustering process, the CPU, memory, communication bandwidth and other parameters are integrated in a measure under. Secondly, with the help of minimum flow and maximum cut theory, the virtual machine cluster is divided and configured to complete the scheduling process of virtual machine to the corresponding physical host. Experimental results show that the virtual machine cluster scheduling based on this new method makes the resource utilization of the physical host higher.
\end{abstract}

Keywords: physical host, virtual machine, scheduling, fusion measure

\section{Introduction}

In order to improve the efficiency and quality of cloud services, virtualization technology is used to remove the coupling between hardware resources and service requirements [1]. Through the use of virtualization technology, the service from the top can be configured in a certain number of virtual machines. Then by finding the right hardware resources to configure the virtual machine, you can complete the cloud services.

The use of virtual machine technology has greatly enhanced the efficiency and flexibility of cloud computing services. In the virtual machine technology research system, the virtual machine deployment is a core issue [2]. Currently, virtual machine deployments are evolving from single virtual machine deployments to virtual machine clusters (multiple associated virtual machines). Deployment strategy relies mainly on resource constraints and load constraints, and according to a certain mathematical model to achieve deployment in the overall best or near best [3]. In order to realize the more convenient virtual machine deployment, based on the sequential deployment strategy, a double-layer scheduling policy was built by Jiang Lan according to the resource constraints of the CPU resource requirements and memory resource requirements of the virtual machine. This virtual machine resources can be dynamically deployed [4]. Based on the background of the cloud environment access of the digital library, Ma Xiao-ting constructed the deployment strategy of the virtual machine from the view of resource restriction. He emphasized the relationship between virtual machine deployment and the diversification of user requirements, and constructed the performance vector to characterize the resource situation, which was the basis for the virtual machine deployment [5]. Zhuang Wei combined the two issues of resource and load, and extracted their properties. He established a multi-attribute analysis strategy to examine the impact of these factors, and then realize the rational allocation of virtual machines [6]. Yang Xing considered the deployment of bulk virtual machine problems. He pointed out that the bulk virtual machine instead of a single virtual machine has become the main research direction of virtual machine deployment technology. It used ant colony optimization algorithm to build a special application for batch virtual machine deployment method, and 
obtained the ideal deployment effect [7]. Starting from the direction of load sensing, Wang Guang-bo constructed the host clustering strategy and virtual machine clustering strategy. He designed the virtual machine cluster deployment process from the perspective of cluster matching. The experimental results confirmed the effectiveness of this method for virtual machine cluster deployment [8].

This paper is also devoted to the study of virtual machine cluster deployment. We start from the two aspects of virtual machine cluster and virtual machine cluster deployment. In the process of the virtual machine cluster deployment, we achieve a more ideal deployment results with the help of graph cut theory.

\section{Method of Virtual Machine Clustering Based on Resource Limitation}

Virtual machine resources with the same or similar attributes are clustered together to form clusters of virtual machines. This is a great convenience for the subsequent configuration of virtual machine resources to the hardware resources. Moreover, this also can greatly improve the efficiency of the entire transport services. Virtual machine clustering is a prerequisite for virtual machine cluster deployment.

In this paper, two kinds of decision conditions are adopted for clustering virtual machine: First, clustered virtual machine resources should have similar hardware resource requirements. Second, the bandwidth requirements for clustered virtual machine resources to communicate with each other should be closer. Using $H_{C P U}$ to represent the virtual machine for CPU hardware resource requirements, $H_{M e m}$ to represent the virtual machine for memory hardware resource requirements and $H_{\text {Hard }}$ to represent the virtual machine hardware resource requirements for hard disk, we can construct the hardware resource demand difference judgment function between two virtual machines, as shown in formula (1):

$$
S_{i, j}(H)=\theta_{1}\left\|H_{C P U}^{i}-H_{C P U}^{j}\right\|+\theta_{2}\left\|H_{M e m}^{i}-H_{M e m}^{j}\right\|+\theta_{3}\left\|H_{\text {Hard }}^{i}-H_{\text {Hard }}^{j}\right\|
$$

Here, $\theta_{1}, \theta_{2}, \theta_{3}$,said the $\mathrm{i}$-th virtual machine and the $\mathrm{j}$-th virtual machine in different hardware resource requirements on the weight of the difference, respectively.

Using $B_{i}$ to represent the bandwidth required for the communication process of the $\mathrm{i}$ th virtual machine, using $B_{j}$ to represent the bandwidth resources required for the $\mathrm{j}$-th virtual machine communication process, we can get the difference judgment function of the two virtual machines on the bandwidth resource demand, as shown in formula (2):

$$
S_{i, j}(B)=\left\|B_{i}-B_{j}\right\|
$$

Based on the similarity judgment function formed by these two types of resource constraints, we construct an overall judgment function for virtual machine clustering, as shown in formula (3).

$$
S_{i, j}=\omega_{1} S_{i, j}(H)+\omega_{2} S_{i, j}(B)
$$

Here, $\omega_{1}, \omega_{2}$ respectively, on behalf of the two categories of resource constraints similarity judgments in the overall judgment function.

By comparing the calculated results with the preset threshold value, we can gather the virtual machines with similar attributes together, and then make the virtual machine cluster through the appropriate communication links. 


\section{Virtual Machine Cluster Deployment Method Based on Graph-Cut Theory}

\subsection{Virtual Machine and Cloud Computing}

The development of virtual machine has hardware acceleration program, it is also because of hard acceleration, but also makes the virtual machine to a wide range of applications, it is also the case, only the concept of cloud computing is brought out, which in turn accelerates the software hardware change. With the increase will affect the qualitative change, people will find that in fact this kind of thing is VM virtualization very inefficient, VMM is actually a personal understanding of the wicked is Yang, he seemed bright actually very real loss Yin reflected, too many unnecessary operating system instances. Operating system is the use of thread / process virtualization to multi task and multi-user operation, each time the system call overhead is very high, so a CPU run multiple operating system instances at the same time, it is a big waste.

The emergence of cloud computing architecture will break this contradiction. Cloud computing is a possible primary global virtual machine scheduling management software framework, but one thing is constantly growing evolution, cloud computing will eventually find its mission, pool, scheduling management and monitoring that is global resources, which is the data center level OS, and things the single OS is exactly the same as. If so, then the AAAS (ApplicationAs a Service) is the cloud computing to achieve the ultimate state, which is equivalent to the opening screen will appear a bunch of points into the application icon, complete your function, exit, end. If the user does not need to IAAS, do not need to directly face the operating system, then engage in so many instances of $\mathrm{VM}$ is actually not necessary, wasting resources.

Cloud computing requires the implementation of a global application level of the scheduling center, rather than scheduling VM. Again consider the big machine why need VM? Because of the big machine that era and has no concept of the cloud computing, xAAS this thinking, you can say that when the software technology is idiotic, very closed and undeveloped, so resource fine-grained segmentation, VM is the Gordian knot of the scheme. We also see that the process level virtual machine (such as LinuxContainer) industry is gradually being concerned. These are the definition of cloud computing software framework, the macro OS, then this will define what is the impact on the hardware? I think that will be spawned two hardware change in the form of a single performance point is above said to be low enough, the intensity should be fine enough, a single point of performance is "enough low, this may surprise people, but in the future it might ah, the nuclear CPU is a good guy; the other is a local multi speed Fabric inter core communication, the CPU/ core can be arbitrary segmentation and combination between them must be in need of a high-speed bus connected with each other, there are a variety of Fabric solutions and products at present, although the relatively low-key but also fairly popular mature, plus silicon optical technologies will Fabric stealth to frame, which provides for a wide range of pool support.

The hardware change is likely to affect the software architecture, the large-scale parallel computing MPI is no longer required remote message passing mechanism, message queue FIFO directly using the Fabric hardware acceleration, will greatly simplify programming for HPC mode can eventually be fully popularized.

\subsection{Virtual Machine Cluster}

When a user sends a request to the cloud, the cloud configures it for virtual machine resources based on the actual situation of the task. In many cases, in order to meet the needs of users and to complete the task with the highest efficiency, it is necessary to clustering virtual machine resources with similar properties, for example, clustering by the method of this paper. When you actually perform a cloud task, the clustered virtual 
machine needs to match the physical resources, that is, how to configure the virtual machine resources to the appropriate host. One physical host can be configured with multiple virtual machines. However, when the performance of the physical host cannot meet the needs of a virtual machine cluster, you need to split this virtual machine cluster reasonably, and then they were configured to a different host. This is the virtual machine cluster deployment.

Given a cluster has been clustered virtual machine structure, as shown in Figure 1.

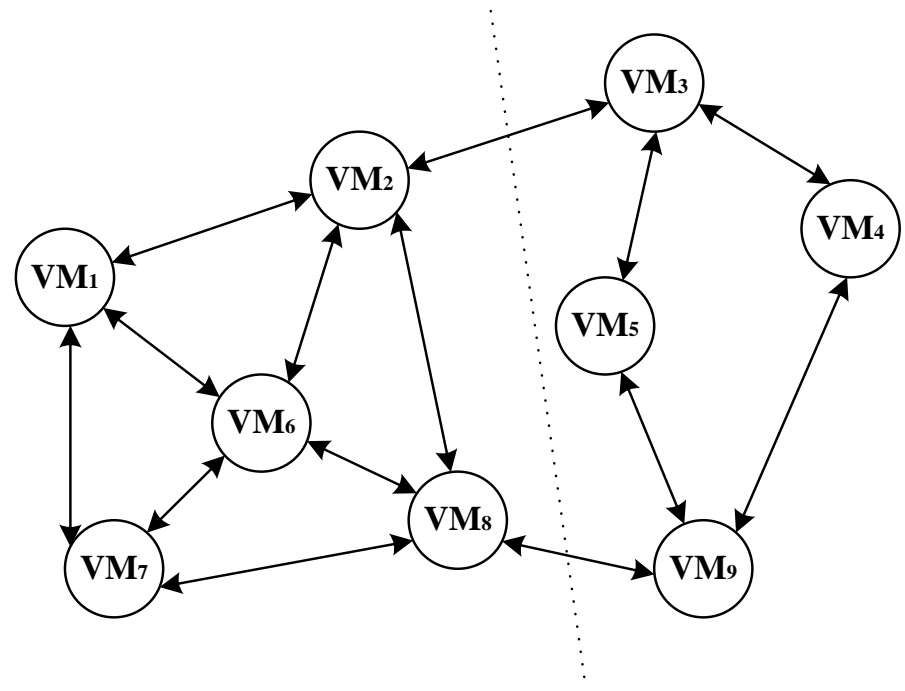

Figure 1. Effect of Multiple Virtual Machine Combinations

This cluster is for the same computing task. However, the configuration of the physical host may not be able to configure the success of a physical host, because the entire cluster of resource demand is too large. At this time, the deployment of this virtual machine cluster has evolved into a segmentation of the virtual machine cluster structure.

From the view of form, the segmentation of virtual machine cluster structure is very similar to that of graph theory. Based on this starting point, we construct the mathematical model of virtual machine cluster according to graph cut theory in order to divide the method.

\subsection{Modeling of Virtual Machine Cluster}

According to the virtual machine cluster structure of Figure 1, each virtual machine is set as the vertex of the cluster network structure, and $V M_{i}$ is used to represent the structure of the virtual machine. It can be shown that the virtual machine cluster in Figure 1 contains 9 vertices.

The properties of the vertex of each virtual machine can be described according to the resources they need. In this way, each attribute of a virtual machine can be expressed in the form of a vector, as shown in the formula (4).

$$
A_{V M_{i}}=\left\{R_{V M_{i}}^{C P U}, R_{V M_{i}}^{\mathrm{Mem}}, R_{V M_{i}}^{\mathrm{Hard}}, R_{V M_{i}}^{B W}\right\}
$$

A bi-directional connection between two virtual machines represents the bandwidth resources required for the virtual machines to communicate with each other. Its properties are described by $B_{V M_{i j}}$.

When configuring an entire virtual machine cluster to a physical host, we must determine the resource cap that the physical host can provide and compare the resource requirements of the cluster with the entire virtual machine cluster. When the physical host 
to provide resources to meet the resource requirements of the entire cluster of virtual machines, direct deployment. Otherwise the virtual machine cluster split deployment. Direct deployment requires the following:

$$
\left\{\begin{aligned}
R_{P M}^{C P U} & >\sum_{i} R_{V M_{i}}^{C P U} \\
R_{P M}^{M e m} & >\sum_{i} R_{V M_{i}}^{M e m} \\
R_{P M}^{\text {Hard }} & >\sum_{i} R_{V M_{i}}^{\text {Hard }} \\
R_{P M}^{B W} & >\sum_{i} R_{V M_{i}}^{B W}+\sum_{j} B_{V M_{i j}}
\end{aligned}\right.
$$

When you need to split the virtual machine cluster in order to redeploy, you can use the principle of graph cut theory to perform the segmentation process. That is, the network in Figure 1 is divided into a series of sub-networks, each sub-network can be found with a matching physical host in order to form a configuration. Here, we do not require that each segmentation form a minimal cut, but rather that the partitioned subnetworks can find the hosts that configure them. From this point of view, and the general map cut method is different.

For a virtual machine cluster, assume that it originally contains $\mathrm{n}$ virtual machines.

After the division formed m clusters, each cluster contains $n_{1}, n_{2}, \cdots, n_{m}$ virtual machines. This $\mathrm{m}$ sub-clusters are arranged in $\mathrm{m}$ physical hosts, the result after the arrangement in accordance with:

$$
\left\{\begin{array}{l}
\forall_{k} R_{P M}^{C P U}>\sum_{i=1}^{n_{k}} R_{V M_{i}}^{C P U} \\
\forall_{k} R_{P M}^{M e m}>\sum_{i=1}^{n_{k}} R_{V M_{i}}^{M e m} \\
\forall_{k} R_{P M}^{\text {Hard }}>\sum_{i=1}^{n_{k}} R_{V M_{i}}^{\text {Hard }} \\
\forall_{k} R_{P M}^{B W}>\sum_{i=1}^{n_{k}} R_{V M_{i}}^{B W}+\sum_{j} B_{V M_{i j}}
\end{array}\right.
$$

\section{Experimental Results and Analysis}

CloudSim is a function library developed in the discrete event simulation package SimJava, because SimJava has some limitations, such as: in the creation of a scalable simulation environment will impose the following restrictions:

(1) it is not allowed to re set the simulation program at run time.

(2) it does not support the creation of a new simulation entity at run time (once the simulation is initialized).

(3) with the increase of system size, the characteristic of SimJava multi thread leads to the increase of performance overhead, and the context switch between too many threads leads to performance degradation.

(4) due to the system debugging, multiple threads bring extra complexity. 
In order to overcome these limitations and can simulate more complex scenes, Australia Melbourne University laboratory grid re design of discrete event framework as shown in figure 1CloudSim core simulation framework class diagram. After re design, CloudSim has the following advantages:

(1) withdrawal of entities (hold).

(2) the context switches in different states (such as waiting for activation). Deactivation and recovery of simulation process.

(3) to create a new entity at run time.

(4) to terminate and restart the simulation run at run time. In Windows and Linux system on cross platform operation, CloudSim inherits the GridSim programming model, support the research and development of cloud computing, and provides the following new features: A: Modeling and simulation infrastructure to support large cloud computing; B: a self-contained support data center, service agent, scheduling the platform and distribution strategy.

In order to verify the effectiveness of the virtual cluster deployment method proposed in this paper, the following experimental research work is carried out. First of all, according to the user's needs, we select the VMs with close attributes from 200 VMs to form clustering clusters, and finally form the clusters of VMs. The communication relationship between virtual machines is shown in Figure 1. The hardware resource requirements of nine virtual machines is shown in Table 1.

Table 1. The Basic Situation of the Virtual Machine in the Experiment

\begin{tabular}{c|c|c|c|c}
\hline Number & CPU & Memory & Hard Disk & Bandwidth \\
\hline VM\#1 & $400 \mathrm{MIPS}$ & $0.8 \mathrm{~GB}$ & $10 \mathrm{~GB}$ & $50 \mathrm{MB} / \mathrm{s}$ \\
\hline VM\#2 & $500 \mathrm{MIPS}$ & $0.8 \mathrm{~GB}$ & $10 \mathrm{~GB}$ & $60 \mathrm{MB} / \mathrm{s}$ \\
\hline $\mathrm{VM} \# 3$ & $400 \mathrm{MIPS}$ & $0.8 \mathrm{~GB}$ & $10 \mathrm{~GB}$ & $50 \mathrm{MB} / \mathrm{s}$ \\
\hline $\mathrm{VM} \# 4$ & $300 \mathrm{MIPS}$ & $0.6 \mathrm{~GB}$ & $15 \mathrm{~GB}$ & $40 \mathrm{MB} / \mathrm{s}$ \\
\hline $\mathrm{VM} \# 5$ & $300 \mathrm{MIPS}$ & $0.6 \mathrm{~GB}$ & $10 \mathrm{~GB}$ & $40 \mathrm{MB} / \mathrm{s}$ \\
\hline $\mathrm{VM} \# 6$ & $500 \mathrm{MIPS}$ & $1.0 \mathrm{~GB}$ & $15 \mathrm{~GB}$ & $60 \mathrm{MB} / \mathrm{s}$ \\
\hline $\mathrm{VM} \# 7$ & $400 \mathrm{MIPS}$ & $1.0 \mathrm{~GB}$ & $15 \mathrm{~GB}$ & $50 \mathrm{MB} / \mathrm{s}$ \\
\hline $\mathrm{VM} \# 8$ & $500 \mathrm{MIPS}$ & $1.0 \mathrm{~GB}$ & $20 \mathrm{~GB}$ & $60 \mathrm{MB} / \mathrm{s}$ \\
\hline $\mathrm{VM} \# 9$ & $400 \mathrm{MIPS}$ & $1.0 \mathrm{~GB}$ & $20 \mathrm{~GB}$ & $50 \mathrm{MB} / \mathrm{s}$ \\
\hline
\end{tabular}

Among them, the virtual machine in addition to the associated virtual machine to communicate with the bandwidth requirements, but also set aside $20 \mathrm{MB} / \mathrm{s}$ bandwidth requirements standby. In this experiment, the communication between each virtual machine requires $10 \mathrm{MB} / \mathrm{s}$ bandwidth. For example, virtual machine \#1 is required to communicate with virtual machines \#2, \#6, \#7, respectively, so that the total bandwidth requirement for $\mathrm{VM} \# 1$ is $50 \mathrm{MB} / \mathrm{s}$.

In the experiment, there are five physical hosts that can be used to deploy virtual machine clusters. The performance parameters are shown in Table 2. 
Table 2. The basic Situation of Physical Host in the Experiment

\begin{tabular}{c|c|c|c|c}
\hline Number & CPU & Memory & Hard Disk & Bandwidth \\
\hline $\begin{array}{c}\text { Physical Host } \\
\# 1\end{array}$ & $2000 \mathrm{MIPS}$ & $2.0 \mathrm{~GB}$ & $200 \mathrm{~GB}$ & $1000 \mathrm{MB} / \mathrm{s}$ \\
\hline $\begin{array}{c}\text { Physical Host } \\
\# 2\end{array}$ & $\begin{array}{c}1500 \mathrm{MIPS}(\text { Dual- } \\
\text { core) }\end{array}$ & $8.0 \mathrm{~GB}$ & $300 \mathrm{~GB}$ & $1000 \mathrm{MB} / \mathrm{s}$ \\
\hline $\begin{array}{c}\text { Physical Host } \\
\# 3\end{array}$ & $\begin{array}{c}1000 \mathrm{MIPS}(\text { Dual- } \\
\text { core) }\end{array}$ & $2.0 \mathrm{~GB}$ & $200 \mathrm{~GB}$ & $1000 \mathrm{MB} / \mathrm{s}$ \\
\hline $\begin{array}{c}\text { Physical Host } \\
\# 4\end{array}$ & $\begin{array}{c}2000 \mathrm{MIPS}(\text { Dual- } \\
\text { core) }\end{array}$ & $8.0 \mathrm{~GB}$ & $500 \mathrm{~GB}$ & $2000 \mathrm{MB} / \mathrm{s}$ \\
\hline $\begin{array}{c}\text { Physical Host } \\
\# 5\end{array}$ & \begin{tabular}{c} 
2000MIPS \\
\hline
\end{tabular} & $4.0 \mathrm{~GB}$ & $300 \mathrm{~GB}$ & $1000 \mathrm{MB} / \mathrm{s}$ \\
\hline
\end{tabular}

After implementing the virtual machine cluster deployment method based on graph cutting theory in this paper, the virtual machine cluster is subdivided into two subclusters, which are respectively configured on the physical host \#2 and the physical host \#5. The schematic diagram is shown in Figure 2.
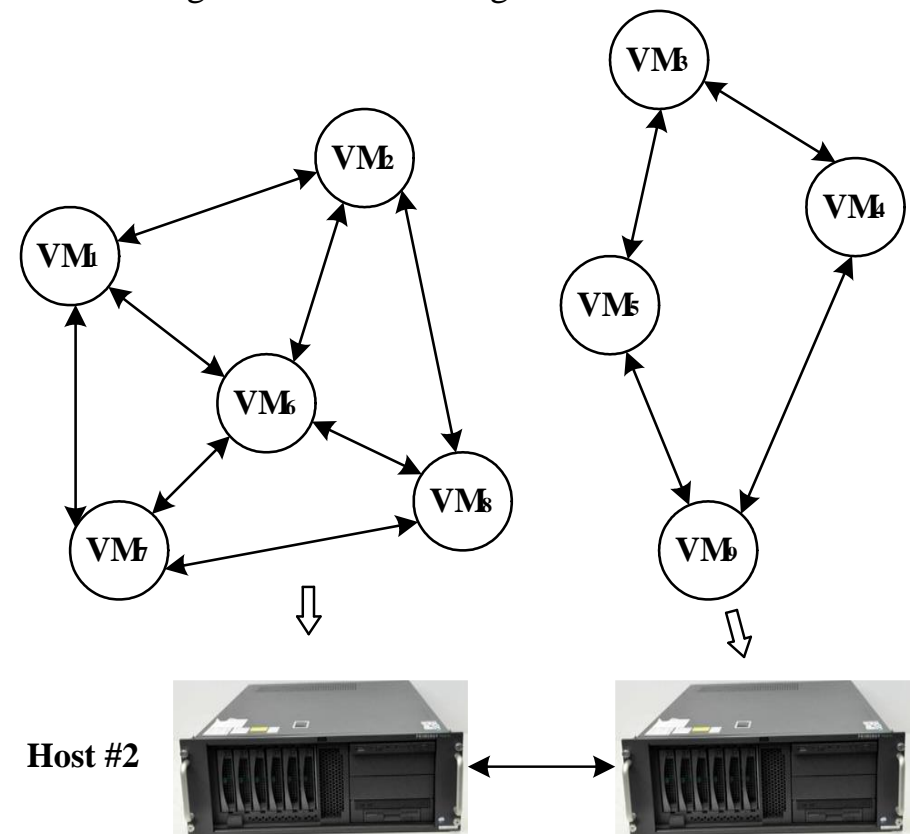

Host \#5

Host \#2

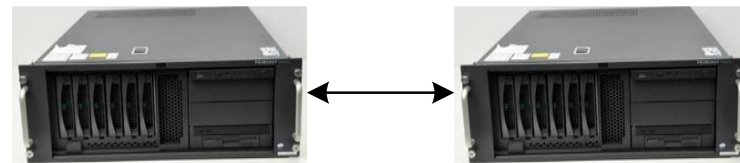

Figure 2. Schematic Diagram of Virtual Machine Scheduling

According to a graph cut segmentation of two virtual machines in a cluster, the first cluster contains VM \#1, VM \#2, VM \#3, VM \#6, VM \#7, and VM \#8, the second cluster contains VM \#3, VM \#4, VM \#5, and VM \#9. The first cluster required a total of 2200MIPS of CPU resources, memory resource requirements of $4.6 \mathrm{~GB}$, hard disk resource requirements of $70 \mathrm{~GB}$, bandwidth requirements of $280 \mathrm{MB} / \mathrm{s}$. The second cluster requires a total CPU resource requirement of 1400 MIPS, a memory resource requirement of $2.4 \mathrm{~GB}$, a hard disk resource requirement of $45 \mathrm{~GB}$, and a bandwidth requirement of $240 \mathrm{MB} / \mathrm{s}$. The hosts that meet the first cluster configuration are physical host \#2 and \#4. The hosts that meet the second cluster configuration are physical host \#2, \#4, and \#5. If the physical host \#4 configured two clusters, there are a lot of resources idle. Therefore, 
the first virtual machine cluster is deployed in the No. 2 host, and the second cluster is deployed in the No. 5 host.

After the original cluster is partitioned, the communications between VM \#2 and VM \#6 and between VM \#8 and VM \#9 will be realized by communication between host \#2 and host \#5.

In order to further verify the efficiency of virtual cluster deployment and single virtual machine deployment, a single virtual machine deployment method based on resource limitation and method based on load balancing were selected as the comparison algorithm. In this way, the bandwidth requirement of the virtual machine deployed on the physical host is investigated. After completing virtual machine deployment in accordance with these three methods, bandwidth demand changes is shown in Figure 3.

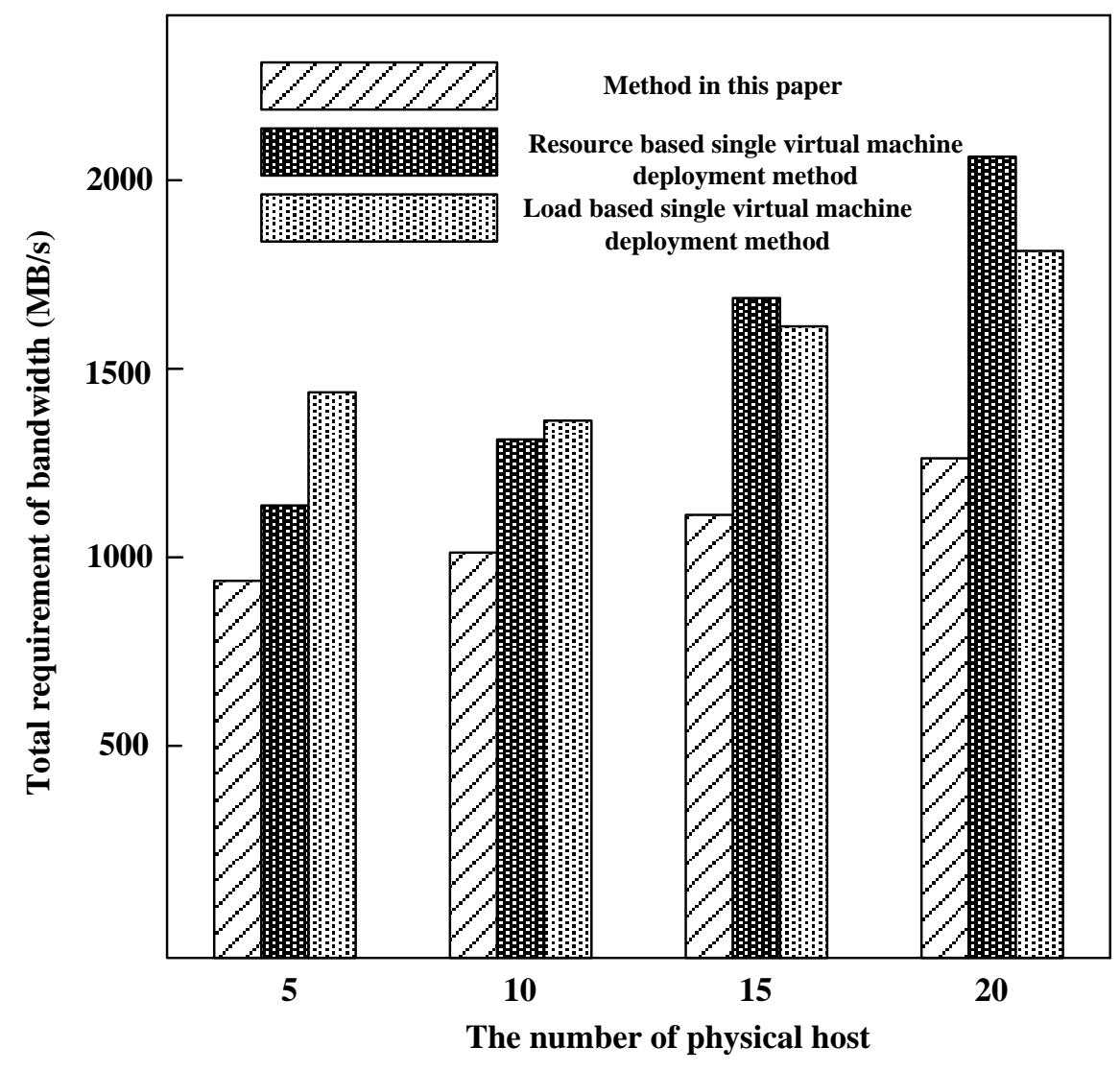

Figure 3. Comparison Results of Resource Utilization

As can be seen from the results in Figure 3, as the number of physical hosts required for virtual machine deployment increases, the total system bandwidth requirements for the three approaches are increasing. We compare the virtual machine cluster deployment method based on graph cut theory in this paper with the two methods of single virtual machine deployment. The results obtained show that the method in this paper not only has a smallest absolute value of the total bandwidth demand, but also the most slow growth trend with the increase of the number of physical hosts.

\section{Conclusion}

With the increasing number of users facing the cloud services, the total user demand will increase, and a single virtual machine deployment strategy will be gradually replaced by virtual machine cluster deployment methods. This paper discusses two core issues in VM cluster deployment: how VMs cluster into clusters, and how the total resource 
requirements of virtual machine clusters exceed physical host resource allocation. For the first problem, this paper proposes a resource-constrained clustering method. Aiming at the second problem, this paper proposes a virtual machine cluster deployment method based on graph cut theory. In virtual cluster deployment, virtual machine cluster network is constructed according to the graph theory, and the virtual machine cluster is partitioned according to the graph cutting theory to complete the reasonable configuration matching the physical host. The experimental results show that the virtual cluster deployment method based on graph cutting theory can obtain reasonable cluster partition and deployment results. Compared with the single virtual machine deployment method, the total bandwidth of the system is smaller, which can save the cloud resources and improve the efficiency of the cloud service.

\section{Acknowledgements}

This study was supported by "Fund project: 2015 open application technology research and project in Harbin (young reserve talented person class A, number: 2015RQQXJ025)".

\section{References}

[1] S. V. Angiuoli, M. Matalka, A. Gussman, K. Galens and M. Vangala, "CloVR: a virtual machine for automated and portable sequence analysis from the desktop using cloud computing", Bmc Bioinformatics, vol. 12, no. 1, (2011), pp. 1-15.

[2] Y. Gao, H. Guan, Z. Qi, Y. Hou and L. Liu, "A multi-objective ant colony system algorithm for virtual machine placement in cloud computing", Journal of Computer \& System Sciences, vol. 79, no. 8, (2013), pp. 1230-1242.

[3] N. Kim, J. Cho and E. Seo, "Energy-credit scheduler: an energy-aware virtual machine scheduler for cloud systems", Future Generation Computer Systems, vol. 32, no. 2, (2014), pp. 128-137.

[4] R. N. Calheiros and R. Ranjan, "CloudSim: a novel framework for modeling and simulation of cloud computing infrastructures and services, Grids-Tr-2009-1", Melbourne Australia: the University of Melbourne, Grid Computing and Distributed Systems Laboratory, (2009).

[5] C Canali and R Lancellotti, "Explpiting ensemble techniques for automatic virtual machine clustering in cloud systems", Automated Software Engineering, vol. 21, no. 21, (2014), pp. 319-344.

[6] M. Armbrust, A. Fox, R. Griffith, A. D. Joseph, R. Katz, A. Konwinski, G. Lee and G. Patterson, "A view of cloud computing", Communications of the ACM, vol. 53, no. 4, (2010), pp. 50-58.

[7] A.Stanik M and H. Kao, "Hardware as a service (HaaS): the completion of the cloud stack", $8^{\text {th }}$ International Conference on Computing Technology and Information Management, (2012), pp. 830-835.

[8] R. Mendel and G. Tal, "Virtual machine monitors: current technology and future trends", IEEE Computer, vol. 38, no. 5, (2005), pp. 39-47. 
International Journal of Grid and Distributed Computing Vol. 9, No. 10 (2016) 